See discussions, stats, and author profiles for this publication at: https://www.researchgate.net/publication/328128220

\title{
Quality not quantity: loneliness subtypes, psychological trauma, and mental health in the US adult population
}

Article in Social Psychiatry and Psychiatric Epidemiology • October 2018 DOI: 10.1007/500127-018-1597-8

\section{CITATIONS}

8 authors, including:

Philip Hyland

13 National University of Ireland, Maynooth 181 PUBLICATIONS 2,242 CITATIONS

SEE PROFILE

Marylene Cloitre

National Center for PTSD

196 PUBLICATIONS 11,600 CITATIONS

SEE PROFILE
READS

5,383

Mark Shevlin

Ulster University

378 PUBLICATIONS 8,776 CITATIONS

SEE PROFILE

Thanos Karatzias

Edinburgh Napier University

153 PUBLICATIONS 2,303 CITATIONS

SEE PROFILE

Some of the authors of this publication are also working on these related projects:

Project CONTEXT (The COllaborative Network for Training and EXcellence in psychoTraumatology) View project

Project ICD-11: PTSD and C-PTSD among former abducted child soldiers in northern Uganda View project 


\title{
Quality not quantity: loneliness subtypes, psychological trauma, and mental health in the US adult population
}

\author{
Philip Hyland ${ }^{2,8}\left(\mathbb{D}^{\circ} \cdot\right.$ Mark Shevlin $^{3} \cdot$ Marylene Cloitre $^{4,5} \cdot$ Thanos Karatzias $^{6,7} \cdot$ Frédérique Vallières $^{2}$. \\ Gráinne McGinty ${ }^{1} \cdot$ Robert Fox $^{8} \cdot$ Joanna McHugh Power ${ }^{1}$
}

Received: 6 July 2018 / Accepted: 18 September 2018

(c) Springer-Verlag GmbH Germany, part of Springer Nature 2018

\begin{abstract}
Purpose Loneliness is a recognised public-health concern that is traditionally regarded as a unidimensional construct. Theories of loneliness predict the existence of subtypes of loneliness. In this study, latent class analysis (LCA) was used to test for the presence of loneliness subtypes and to examine their association with multiple mental health variables.

Methods A nationally representative sample of US adults $(N=1839)$ completed the De Jong Gierveld Loneliness Scale, along with self-report measures of childhood and adulthood trauma, psychological wellbeing, major depression, and generalized anxiety.

Results When treated as a unidimensional construct, $17.1 \%$ of US adults aged 18-70 were classified as lonely. However, the LCA results identified four loneliness classes which varied quantitatively and qualitatively: 'low' (52.8\%), 'social' (8.2\%), 'emotional' (26.6\%), and 'social and emotional' (12.4\%) loneliness. The 'social and emotional' class were characterised by the highest levels of psychological distress, followed by the 'emotional' class. The 'social' loneliness class had similar mental health scores as the 'low' loneliness class. Childhood and adulthood trauma were independently related to the most distressed loneliness classes.

Conclusions Current findings provide support for the presence of subtypes of loneliness and show that they have unique associations with mental health status. Recognition of these subtypes of loneliness revealed that the number of US adults aged 18-70 experiencing loneliness was twice as high as what was estimated when loneliness was conceptualized as a unidimensional construct. The perceived quality, not the quantity, of interpersonal connections was associated with poor mental health.
\end{abstract}

Keywords Loneliness · Latent class analysis · Mental health

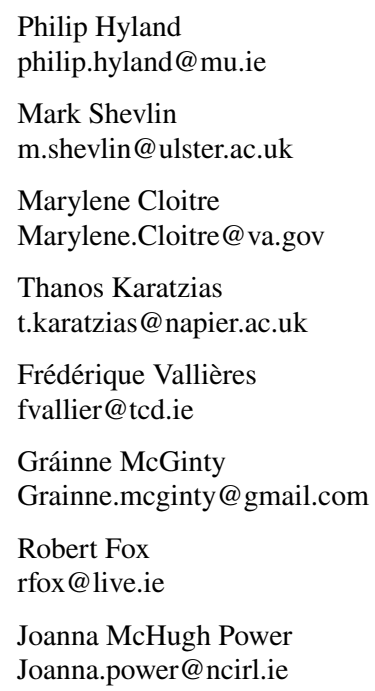

1 School of Business, National College of Ireland, Dublin, Ireland

2 Centre for Global Health, School of Psychology, Trinity College Dublin, Dublin, Ireland

3 School of Psychology, Ulster University, Derry, Northern Ireland, UK

4 National Center for PTSD, Veterans Affairs Palo Alto Health Care System, Palo Alto, CA, USA

5 Department of Psychiatry and Behavioral Science, Stanford University, Palo Alto, CA, USA

6 School of Health and Social Care, Edinburgh Napier University, Edinburgh, UK

7 Rivers Centre for Traumatic Stress, NHS Lothian, Edinburgh, UK

8 School of Psychology, Maynooth University, Kildare, Ireland 


\section{Introduction}

Loneliness is increasingly recognised as a global health concern [1], and is known to be correlated with, and predictive of, psychological and physical disorders [2, 3]. The number of people experiencing loneliness varies across nations. Prevalence rates of loneliness in nine former Soviet Union countries ranged from $4.4 \%$ (Azerbaijan) to $17.9 \%$ (Moldova) [4]. In a nationally representative sample of Danish adults, $21 \%$ of people reported being either moderately (16.4\%) or severely (4.6\%) lonely [5]. In Quebec, $14.5 \%$ of individuals aged 15 years and older reported loneliness [6]. No study has yet examined the prevalence rates of loneliness amongst the adult population of the United States (US); however, a nationally representative survey of US adults aged 45 years and older found that $35 \%$ reported loneliness [7]. The relatively high rate of loneliness in this US study was likely due to the use of an older adult sample given that loneliness rises substantially in older age [5]. Determining the prevalence rate of loneliness is exceptionally challenging as there is no established diagnostic algorithm for classifying loneliness. Moreover, variation in the methods used to measure loneliness (single-item vs. multiple-item scales) and to classify individuals as "being lonely" (a certain response option for a single-item measure or use of a given cut-off score for multi-item scales) is likely to lead to considerable variation in estimates of the prevalence rates of loneliness.

Loneliness is typically treated as a unidimensional construct, and consequently, prevalence rates of loneliness tends to be determined based on whether or not an individual exceeds a total score [e.g., 5-7]. However, many have challenged the assumption that loneliness is a unidimensional construct and have instead argued that multiple types of loneliness exist [8]. Weiss' [9] multidimensional theory of loneliness, for example, distinguishes between 'social' (deficiencies of social integration) and 'emotional' (deficiencies of close attachments) loneliness. Factor analytic studies indicate that measurement models which distinguish between these dimensions of loneliness are superior to unidimensional models $[10,11]$, and that social and emotional loneliness are only moderately correlated [12]. Failure to recognise naturally occurring subtypes of loneliness may, therefore, lead to unreliable estimates of the prevalence rate of loneliness.

Further support for the existence of subtypes of loneliness comes from studies indicating distinct antecedents of social and emotional loneliness. Social loneliness has been shown to be related to reductions in social network size, whereas emotional loneliness has been shown to be related to deficits in intimate partner relationships [13]. Additionally, males tend to display higher social and lower emotional loneliness, while females show the opposite pattern. Social and emotional loneliness also share similar risk-correlates such as partnership status, increasing age, low subjective wellbeing, widowhood, and lower levels of self-esteem [10, 13]. Childhood and adulthood traumatization have both been linked to an increased likelihood of experiencing loneliness [14-18], and loneliness has been shown to mediate the relationship between traumatic exposure and psychiatric morbidity [19]. No study has yet investigated the relationship between loneliness and childhood and adulthood trauma simultaneously, and more importantly, no study has yet examined if the developmental timing of traumatic exposure is differentially associated with proposed subtypes of loneliness. The existing literature is also inconclusive regarding the relationship between loneliness subtypes and mental health status. For example, some studies have found depression and anxiety to be associated with social loneliness [20, 21]; others have found depression to be more strongly associated with emotional loneliness [21-23]; and yet others show that depression is similarly related to social and emotional loneliness [24].

The inconsistent findings are likely due to multiple factors including variation in the measurement of loneliness, the use of non-representative samples, and imprecise methods of classifying loneliness subtypes. Traditionally, purported subtypes of loneliness are represented by summed subscale scores from measures of loneliness, and these subscales are known to be moderately correlated [12]. This method does not discriminate between different types of loneliness and leaves results vulnerable to the effects of multicollinearity. The application of latent class analysis (LCA) offers a methodologically rigorous approach to (1) determining if unique subtypes of loneliness exist, and (2) if so, isolating these subtypes through the construction of non-overlapping, homogeneous classes of individuals (e.g., 'emotionally lonely' individuals and 'socially lonely' individuals). To date, however, only one study has used LCA methods to determine if distinct subtypes (or latent classes) of loneliness exist [25]. In this study of Northern Irish adolescents who completed the UCLA-Loneliness Scale [26], four distinct loneliness classes were identified. The classes differed quantitatively ('low', 'moderate', and 'high' loneliness classes) and qualitatively (one class was characterised by high levels of 'social loneliness'). Moreover, the classes were also found to significantly differ in relation to their risk of psychiatric morbidity.

Given the possible therapeutic and prevention implications of identifying naturally occurring loneliness subtypes in the population, as well as the extant methodological limitations in this field of research, the current study, based on a nationally representative sample of US adults aged 18-70 years, was performed to investigate five objectives: 
1. To determine the prevalence rate of loneliness in the US adult population aged 18-70 years using a standard method employed in the literature when loneliness is conceptualised as a unidimensional construct.

2. Using LCA techniques, we examined if qualitatively distinct subtypes of loneliness existed as predicted by Weiss' [9] multidimensional theory of loneliness (i.e., 'social' and 'emotional' loneliness). We predicted that multiple latent classes of loneliness would be identified. Loneliness classes that differed on purely quantitative grounds (e.g., 'high', 'medium', and 'low' loneliness classes) would falsify the hypothesis that subtypes of loneliness exist. Evidence of qualitatively distinct classes (e.g., classes that have similar levels of loneliness but are markedly distinct in their profile of loneliness) would support the hypothesis that subtypes of loneliness exist.

3. We examined if loneliness subtypes were differentially related to psychological wellbeing, major depressive disorder (MDD), and generalized anxiety disorder (GAD).

4. We examined if specific relationships existed between loneliness subtypes and antecedent risk-factors including childhood and adulthood traumatization.

5. We investigated if the relationships between childhood and adulthood traumatization and psychological wellbeing, MDD, and GAD, respectively, were influenced by the specific subtype of loneliness that one was characterised by.

\section{Methods}

\section{Participants and procedures}

This study used a nationally representative household sample of non-institutionalised adults currently residing in the United States. Data were collected in March 2017 using an online research panel randomly recruited through probability-based sampling. To be included in the current study, respondents had to be aged between 18 and 70 years at the time of the survey, and have experienced at least one traumatic event in their lifetime. A total of 3953 participants were screened to meet the inclusion criteria and a total of 1839 people qualified as valid cases (eligibility rate $=46.3 \%$ ). The survey design oversampled among females and minority populations (African American and Hispanic), each at a 2:1 ratio. To adjust for this oversampling, and to ensure the nationally representative nature of the sample, the data were weighted to be representative of the entire US adult population aged 18-70 years. All selfreport surveys were completed on-line and the median time of completion was $18 \mathrm{~min}$. Individuals received no payment for participation, but were incentivised to participate through entry into a raffle for prizes. The study received ethical approval from the Research Ethics committee of the institution to which the first author is affiliated.

The mean age of the weighted sample was 44.55 years $(\mathrm{SD}=14.89)$ and included a similar number of males $(48 \%$, $n=883)$ and females $(52 \%, n=956)$. The majority of the sample was married $(55.3 \%, n=1016)$ and $8.1 \%(n=149)$ indicated that they were co-habiting with a partner. These individuals were subsequently combined to reflect a group that were 'in a relationship'. The remainder of the sample indicated that they were single $(23.3 \%, n=428)$, divorced $(10.9 \%, n=202)$, or widowed $(2.4 \%, n=44)$. These individuals were combined to reflect a group that were "not in a relationship'. The majority of the sample were 'White, Non-Hispanic' ( $63.8 \%, n=1173)$, followed by 'Hispanic' (16.9\%, $n=310)$, 'Black, Non-Hispanic' $(11.8 \%, n=217)$, 'Other, Non-Hispanic' $(6.3 \%, n=115)$, and ' $2+$ Races, Non-Hispanic' $(1.3 \%, n=24)$. Approximately one-third of the sample reported that their highest level of educational achievement was a 'Bachelor's degree or higher' (31.8\%, $n=585$ ), while similar amounts indicated 'some college' (30.3\%, $n=558)$, or 'finishing high school' $(28.7 \%, n=528)$, and $9.1 \%(n=168)$ indicated that they 'did not finish high school'. Nearly half of the sample earned US $\$ 75,000$ or more per year $(48.5 \%, n=891), 29.8 \%(n=547)$ earned between US $\$ 35,000$ and US $\$ 74,999$ per year, $11.0 \%(n=202)$ earned between US $\$ 20,000$ and US $\$ 34,999$ per year, and 10.8\% $(n=199)$ earned between US\$0-US $\$ 19,999$ per year.

\section{Measures}

\section{Loneliness}

The six-item De Jong Gierveld Loneliness Scale [27] was used to measure feelings of social and emotional loneliness, each measured by three items. The emotional loneliness items are phrased in a negative manner and the social loneliness items are phrased in a positive manner. All items were answered using a three-point Likert scale of 'Very much agree' (1), 'Somewhat agree' (2), and 'Do not agree' (3). Following the scoring guidelines provided by the scale authors [27], all items were dichotomised to reflect the 'presence' (1) or 'absence' (0) of an indicator of loneliness. For the emotional loneliness items, agreement responses were taken to indicate item endorsement, while for the social loneliness items, disagreement responses were taken to indicate item endorsement. This measure has been shown to be reliable and valid in large-scale general population surveys [28]. The internal reliability (Cronbach's alpha) of the full scale $(\alpha=0.81)$ and the 'social' $(\alpha=0.88)$ and 'emotional' $(\alpha=0.74)$ subscales were satisfactory within the current sample. There is no agreed upon cut-off score for the six-item De Jong Gierveld Loneliness Scale to identify 
loneliness cases. In the current study, we followed the recommendations of Shevlin et al. [29] that caseness for loneliness should be determined by selecting only those individuals with a score 1 standard deviation above the sample mean.

\section{Childhood and adulthood traumatic exposure}

A modified version of the Life Events Checklist for DSM-5 [30] was used to measure traumatic exposure during childhood and adulthood. Individuals answered on a 'Yes' (1) or 'No' $(0)$ basis if they had experienced any of 14 common traumatic events 'before the age of 18' (childhood) or 'at or after the age of 18' (adulthood). Three items from the Adverse Childhood Experiences questionnaire [31] assessing physical abuse, sexual abuse, and neglect were also used to supplement the measurement of childhood trauma. Summed total scores of childhood (0-17) and adulthood $(0-14)$ trauma were calculated.

\section{Psychological wellbeing}

Psychological wellbeing was assessed using the five-item World Health Organization Well-Being Index (WHO-5) [32]. The WHO-5 is an internationally validated measure of positive psychological health. A recent review of 213 international studies supported the reliability and validity of the scale [33]. Respondents are asked to indicate how they have been feeling over the past 2 weeks to each positively phrased statement along a six-point Likert scale ranging from 'At no time' (0) to 'All of the time' (5). Scores range from 0 to 25 , with higher scores reflecting greater psychological wellbeing. Scores $\leq 13$ are indicative of poor mental health and the possible presence of a psychiatric disorder [34]. The reliability of the WHO-5 among the current sample was high $(\alpha=0.93)$.

\section{Major depressive disorder (MDD) and generalized anxiety disorder (GAD)}

Symptoms of MDD and GAD were measured using the eight-item Patient Health Questionnaire Depression Scale (PHQ-8) [35] and the Generalized Anxiety Disorder 7-item Scale (GAD-7). These scales assess the symptoms of MDD and GAD in-line with DSM-5 criteria (the PHQ-8 excludes one item reflecting the suicidality/self-harm symptom for MDD). For both measures respondents indicate how often they have been bothered by each symptom over the last 2 weeks using a four-point Likert scale ranging from 'Not at all' (0) to 'Nearly every day' (3). Scores on the PHQ-8 range from 0 to 24 and scores on the GAD-7 range from 0 to 21 . In both cases, higher scores reflect greater symptomatology, and scores $\geq 10$ are considered indicative of diagnostic status [35, 36]. The PHQ-8 [37] and the GAD-7 [38] have demonstrated excellent psychometric properties. The internal reliability of the PHQ-8 $(\alpha=0.93)$ and the GAD-7 $(\alpha=0.94)$ were excellent within the current sample.

\section{Data analysis}

The analytic process for the current study included three linked phases and all analyses were conducted using Mplus 7.4 [39]. First, LCA was performed based on binary responses to the six De Jong Gierveld Loneliness Scale items so as to determine the optimal number of latent classes of loneliness. The fit of six models (1-6 classes) were assessed and all models were estimated using robust maximum likelihood [40]. Missing data were low (1.5\%) and the models were estimated using all available information. To avoid solutions based on local maxima, 500 random sets of starting values were used followed by 100 final stage optimizations. The relative fit of the latent class models were compared using three information theory based fit statistics: the Akaike information criterion (AIC) [41], the Bayesian information criterion (BIC) [42] and the samplesize-adjusted BIC (ssaBIC) [43]. The model that produces the lowest value on each criterion can be judged to be best. Additionally, the Lo-Mendell-Rubin adjusted likelihood ratio test (LMR-A) [44] was used to compare models with increasing numbers of latent classes, whereby a non-significant value suggests that the model with one less class should be accepted. Evidence from simulation studies indicates that the BIC is the best index to identify the correct number of latent classes [45].

Second, mean differences on the mental health variables (psychological wellbeing, MDD, and GAD) were compared across the identified latent classes. To avoid shifts in the latent classes due to the inclusion of auxiliary variables, an automatic Bolck-Croon-Hagenaars (BCH) method [46] was implemented. The $\mathrm{BCH}$ method has been shown in simulation studies to outperform alternative approaches such as the '3-step method' or the 'Lanza method' [47, 48]. The $\mathrm{BCH}$ method overcomes the primary limitation of the 3-step method (shifting latent classes as a result of the inclusion of auxiliary variables) due to the fact that it "uses a weighted multiple group analysis, where the groups correspond to the latent classes, and thus the class shift is not possible because the classes are known" [49, p. 2]. Additionally, unlike the Lanza method, the $\mathrm{BCH}$ method does not require homogeneity of variance for the auxiliary variables.

Third, a manual BCH method [49] was conducted to evaluate: (1) the unique associations between five covariates (age, sex, relationship status, childhood trauma, and adulthood trauma) and class membership; and (2) class-specific associations between these covariates and psychological wellbeing, MDD, and GAD. This manual $\mathrm{BCH}$ process is completed in two steps. In the first step, the latent class 
measurement model is estimated and the $\mathrm{BCH}$ class weights are saved. In the second step, the general auxiliary model is evaluated. In this case, the latent classes were (1) simultaneously regressed on all covariates, and (2) the mental health variables were simultaneously regressed on all covariates conditional on the latent class variable. This analytical process allows for the effect of each covariate on class membership to be determined without any shift in the latent classes, and for the class-specific relationships between the covariates and the mental health variables to be determined simultaneously.

\section{Results}

\section{Objective 1-prevalence rate of loneliness in the US adult population when treated as a unidimensional construct}

The mean score for the six-item De Jong Gierveld Loneliness Scale was $1.76(\mathrm{SD}=1.77)$. A total of $17.1 \%(n=307)$ of the sample had a mean score of loneliness greater than 1 SD above the sample mean and were, therefore, classified as lonely.

\section{Objective 2 -LCA results}

The BIC and ssaBIC results were lowest for the four-class solution, suggesting its statistical superiority, however, the LMR-A became non-significant at four-classes suggesting the superiority of a three-class solution. Based on the simulation work of Nylund et al. [44] which indicated that the BIC is the best method for determining the optimal class solution, along with the interpretability of the different class solutions, it was determined that the four-class model was the best representation of the latent class structure of loneliness. The profile plot of the four-class solution is presented in Fig. 1 and all fit indices for the LCA are presented Table 1.

Class 1 was the largest $(52.8 \%, n=984)$ and was characterised by low probabilities of endorsing each loneliness item. This class was labelled the 'low loneliness' class. Class 2 was the smallest $(8.2 \%, n=138)$ and was characterised by low probabilities of endorsing the emotional loneliness items and high probabilities of endorsing the social loneliness items. This class was labelled the 'social loneliness' class. Class $3(26.6 \%, n=472)$ was characterised by high probabilities of endorsing the emotional loneliness items and low probabilities of endorsing the social loneliness items.

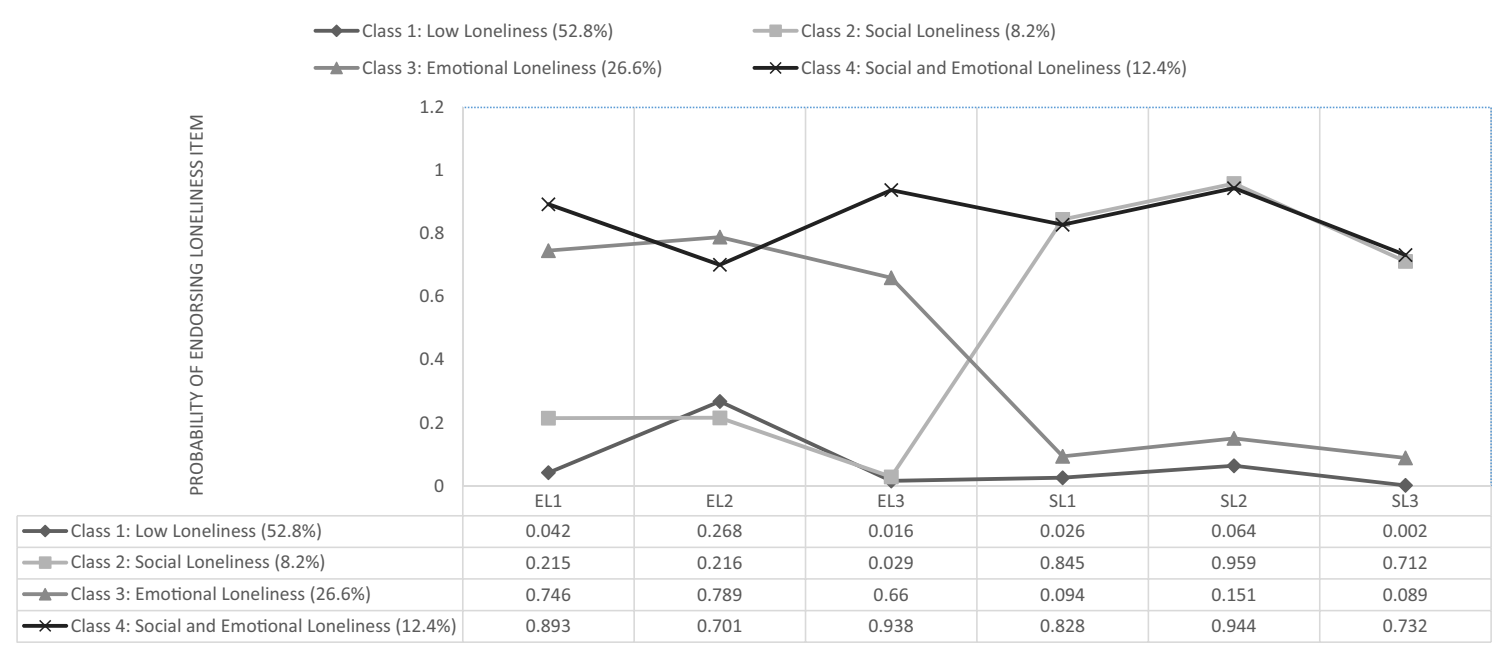

Fig. 1 Latent class profile of loneliness

Table 1 LCA fit statistics based on responses to the De Jong Gierveld Loneliness Scale $(N=1815)$

\begin{tabular}{lllllll}
\hline Classes & Log likelihood & AIC & BIC & ssaBIC & LMR-A $(p)$ & Entropy \\
\hline 1 & -6350 & 12,712 & 12,745 & 12,726 & - & - \\
2 & -5464 & 10,955 & 11,027 & 10,986 & $1737(<0.001)$ & 0.84 \\
3 & -5156 & 10,352 & 10,462 & 10,399 & $605(<0.001)$ & 0.82 \\
$\mathbf{4}$ & $-\mathbf{5 0 5 7}$ & $\mathbf{1 0 , 1 6 9}$ & $\mathbf{1 0 , 3 1 7}$ & $\mathbf{1 0 , 2 3 1}$ & $\mathbf{1 9 4}(\mathbf{0 . 2 0 3})$ & $\mathbf{0 . 8 3}$ \\
5 & -5042 & 10,153 & 10,340 & 10,232 & $29(0.415)$ & 0.87 \\
6 & -5031 & 10,144 & 10,370 & 10,240 & $22(0.395)$ & 0.87 \\
\hline
\end{tabular}

Best-fitting model in bold 
This class was labelled the 'emotional loneliness' class. Finally, class $4(12.4 \%, n=222)$ was characterised by high probabilities of endorsing all loneliness items. This class was labelled the 'social and emotional loneliness' class.

\section{Objective 3-class differences on mental health variables}

There were statistically significant overall differences between the classes on psychological wellbeing, MDD, and GAD, and all pairwise comparisons between the latent classes were statistically significant (see Table 2 ). The pattern of results was similar across all mental health variables. There was a clear gradient of psychological distress across classes with the 'low loneliness' class the least distressed, followed by the 'social loneliness' class, then the 'emotional loneliness' class, and then the 'social and emotional loneliness' class being the most distressed. These results indicate that while the experience of social loneliness is associated with slight diminutions in overall mental health, relative to the low loneliness class, the experience of emotional loneliness has a substantially greater, and more negative impact on overall mental health status. Furthermore, the combination of social and emotional loneliness is associated with the poorest mental health status.

\section{Objective 4-correlates of class membership}

Table 3 reports the results of a multinomial logistic regression analysis assessing the unique associations between class membership and each covariate. Compared to the 'low loneliness' class, membership of the 'social loneliness' class was significantly associated with younger age. Membership of the 'emotional loneliness' class was significantly associated with younger age, being female, not being in a relationship, and an increased number of childhood traumas.
Table 2 Tests of differences of means (standard errors) across loneliness classes $(N=1815)$
Table 3 Correlates of class membership based on results of a multinomial logistic regress analysis $(N=1772)$

\begin{tabular}{llll}
\hline & Psychological wellbeing & Depression & Generalized anxiety \\
\hline Class 1: Low loneliness & $18.20(0.18)$ & $1.17(0.10)$ & $1.23(0.10)$ \\
Class 2: Social loneliness & $15.93(0.89)$ & $2.78(0.62)$ & $2.48(0.45)$ \\
Class 3: Emotional loneliness & $11.96(0.39)$ & $7.06(0.38)$ & $6.06(0.34)$ \\
Class 4: Social and emotional loneliness & $7.10(0.48)$ & $10.64(0.63)$ & $8.96(0.58)$ \\
Overall test ${ }^{\mathrm{a}}\left(\right.$ Wald $\left.\chi^{2}\right)$ & $618.19^{* * *}$ & $463.14 * * *$ & $357.05^{* * *}$ \\
Pairwise tests ${ }^{\text {b }}$ (Wald $\left.\chi^{2}\right)$ & & & \\
Class 1 vs. 2 & $6.24^{*}$ & $6.61^{*}$ & $7.34^{*}$ \\
Class 1 vs. 3 & $192.40^{* *}$ & $211.94^{* *}$ & $169.53^{* *}$ \\
Class 1 vs. 4 & $480.21^{* *}$ & $225.55^{* *}$ & $172.38^{* *}$ \\
Class 2 vs. 3 & $16.52^{* *}$ & $34.51^{* *}$ & $40.06^{* *}$ \\
Class 2 vs. 4 & $71.31^{* *}$ & $74.18^{* *}$ & $72.35^{* *}$ \\
Class 3 vs. 4 & $57.29^{* *}$ & $21.89^{* *}$ & $17.00^{* *}$ \\
\hline
\end{tabular}

Statistical significance $=* * p<0.001, * p<0.01$

${ }^{a}$ All tests have 3 degrees of freedom

${ }^{\mathrm{b}}$ All tests have 1 degree of freedom

\begin{tabular}{lllc}
\hline & $\begin{array}{l}\text { Class 2: Social loneliness } \\
B(\mathrm{SE})[\mathrm{OR}]\end{array}$ & $\begin{array}{l}\text { Class 3: Emotional loneliness } \\
B(\mathrm{SE})[\mathrm{OR}]\end{array}$ & $\begin{array}{l}\text { Class 4: Social and } \\
\text { emotional loneliness } \\
B \text { (SE) [OR] }\end{array}$ \\
\hline Age & $-0.03(0.01)^{* *}[0.97]$ & $-0.02(0.01)^{* *}[0.98]$ & $-0.03(0.01)^{* *}[0.97]$ \\
Sex & $-0.21(0.25)[0.81]$ & $0.59(0.18)^{* *}[1.80]$ & $0.62(0.22)^{*}[1.86]$ \\
Relationship & $-0.17(0.29)[0.84]$ & $0.64(0.18)^{* *}[1.90]$ & $0.42(0.22)[1.52]$ \\
Adult trauma & $0.09(0.07)[1.09]$ & $0.04(0.06)[1.04]$ & $0.16(0.06)^{*}[1.17]$ \\
Child trauma & $0.08(0.07)[1.08]$ & $0.25(0.05)^{* *}[1.28]$ & $0.23(0.06)^{* *}[1.26]$ \\
\hline
\end{tabular}

Reference group for all analyses if Class 1 (the 'Low Loneliness' class)

Sex is scored $(0=$ male, $1=$ female $)$; relationship status is scored $(0=$ married or in a relationship, $1=$ widowed, divorced, or single)

$B$ unstandardized beta value, $S E$ standard error, $O R$ odds ratio

Statistical significance $=* p<0.01, * * p<0.001$ 
Membership of the 'social and emotional loneliness' class was significantly associated with younger age, being female, an increased number of childhood traumas, and an increased number of adulthood traumas.

\section{Objective 5-class-specific associations between covariates and mental health variables}

The results of the class-specific associations between each covariate and each mental health variable are presented in Table 4. In the 'low loneliness' class, the model explained almost no variance in each of the mental health variables. Adulthood trauma was significantly associated with poorer psychological wellbeing, and higher levels of MDD and GAD. Additionally, being female was significantly associated with increased levels of MDD and GAD. In the 'social loneliness' class, the model explained $>10 \%$ of variance in each mental health variable, and increased frequency of adulthood trauma was significantly and positively associated with MDD and GAD scores. In the 'emotional loneliness' class, the model explained $>20 \%$ of variance in MDD and GAD scores, and $<10 \%$ of variance in psychological wellbeing scores. Increased frequency of childhood trauma was significantly associated with lower levels of psychological wellbeing, and higher levels of MDD and GAD. Finally, in the 'social and emotional loneliness' class, the model explained a robust percentage of variance in MDD $(27 \%)$ and GAD (35\%) scores, but substantially less variance in psychological wellbeing (6\%) scores. Increased frequency of adulthood trauma was significantly associated with psychological wellbeing and MDD scores; being female was significantly associated with increased levels of MDD and
Table 4 Class-specific association between each covariate and all mental health variables $(N=1772)$

\begin{tabular}{|c|c|c|c|}
\hline & $\begin{array}{l}\text { Psychological wellbeing } \\
\beta \text { (SE) }\end{array}$ & $\begin{array}{l}\text { Depression } \\
\beta \text { (SE) }\end{array}$ & $\begin{array}{l}\text { Generalized anxiety } \\
\beta(\mathrm{SE})\end{array}$ \\
\hline \multicolumn{4}{|c|}{ Class 1: Low loneliness (52.8\%) } \\
\hline Age & $0.03(0.04)$ & $-0.00(0.03)$ & $-0.04(0.03)$ \\
\hline Sex & $-0.05(0.04)$ & $0.07(0.02)^{* *}$ & $0.10(0.03)^{* * *}$ \\
\hline Relationship status & $0.01(0.04)$ & $-0.03(0.03)$ & $0.00(0.03)$ \\
\hline Adult trauma & $-0.15(0.05)^{* * *}$ & $0.09(0.04)^{*}$ & $0.08(0.04)^{*}$ \\
\hline Childhood trauma & $0.08(0.05)$ & $0.01(0.04)$ & $-0.00(0.04)$ \\
\hline$R^{2}$ & 0.02 & 0.01 & 0.02 \\
\hline \multicolumn{4}{|c|}{ Class 2: Social loneliness (8.2\%) } \\
\hline Age & $-0.21(0.12)$ & $0.07(0.08)$ & $0.05(0.08)$ \\
\hline Sex & $0.05(0.16)$ & $-0.08(0.14)$ & $-0.05(0.11)$ \\
\hline Relationship status & $0.13(0.15)$ & $-0.14(0.12)$ & $-0.14(0.10)$ \\
\hline Adult trauma & $-0.27(0.19)$ & $0.29(0.12)^{* *}$ & $0.30(0.11)^{* *}$ \\
\hline Childhood trauma & $0.04(0.18)$ & $-0.02(0.15)$ & $-0.05(0.14)$ \\
\hline$R^{2}$ & 0.17 & 0.11 & 0.11 \\
\hline \multicolumn{4}{|c|}{ Class 3: Emotional loneliness (26.6\%) } \\
\hline Age & $-0.07(0.08)$ & $0.08(0.09)$ & $-0.11(0.08)$ \\
\hline Sex & $-0.15(0.08)$ & $0.13(0.09)$ & $0.22(0.08)$ \\
\hline Relationship status & $0.11(0.07)$ & $0.03(0.08)$ & $-0.06(0.08)$ \\
\hline Adult trauma & $-0.01(0.11)$ & $0.12(0.13)$ & $0.12(0.14)$ \\
\hline Childhood trauma & $-0.17(0.05)^{*}$ & $0.33(0.10)^{* * *}$ & $0.35(0.10)^{* * *}$ \\
\hline$R^{2}$ & 0.08 & 0.21 & 0.25 \\
\hline \multicolumn{4}{|c|}{ Class 4: Social and emotional loneliness (12.4\%) } \\
\hline Age & $0.00(0.08)$ & $-0.28(0.11)$ & $-0.34(0.10)^{* * *}$ \\
\hline Sex & $-0.15(0.11)$ & $0.33(0.15)^{*}$ & $0.38(0.14)^{* *}$ \\
\hline Relationship status & $0.01(0.10)$ & $-0.05(0.14)$ & $-0.03(0.13)$ \\
\hline Adult trauma & $-0.23(0.09)^{* *}$ & $0.38(0.15)^{* *}$ & $0.28(0.17)$ \\
\hline Childhood trauma & $0.03(0.10)$ & $0.08(0.20)$ & $0.21(0.19)$ \\
\hline$R^{2}$ & 0.06 & 0.27 & 0.35 \\
\hline
\end{tabular}

Sex is scored $(0=$ male, $1=$ female $)$; Relationship status is scored $(0=$ married or in a relationship, $1=$ widowed, divorced, or single)

$\beta$ standardized beta value, $S E$ standard error, $O R$ odds ratio Statistical significance $={ }^{*} p<0.05, * * p<0.01, * * * p<0.001$ 
GAD; and younger age was significantly associated with higher levels of GAD.

\section{Discussion}

Loneliness is typically treated as a unidimensional construct and prevalence rates have been derived from this conceptualization [4-7]. However, theoretical models and empirical data suggest that loneliness may in fact be multidimensional in nature [8-12], and if so, prevalence estimates are likely to be in error. Moreover, empirical findings regarding the risk-factors for loneliness are also likely to be in error if the construct is not conceptualised in an accurate manner. The objective of this study was to investigate whether subtypes of loneliness were identifiable within a nationally representative sample of US adults aged 18-70; and if so, to determine how recognition of loneliness subtypes would influence the prevalence rate of loneliness, as well as the associations with risk-factors and mental health variables.

Using a typical method employed in the literature for determining prevalence rates when loneliness is treated as a unidimensional construct [29], we found that $17.1 \%$ of US adults aged 18-70 would have been classified as experiencing loneliness. This finding is generally consistent with population prevalence rates from similarly aged representative samples from Quebec (14.0\%), Denmark (21.0\%), Armenia (10.7\%), Belarus (8.9\%), Georgia (12.3\%), Moldova (17.9\%), and Ukraine (10.8\%) [4-6]. However, the LCA results indicated that loneliness was not unidimensional in nature. Two of the four classes, the 'social' and 'emotional' loneliness classes, differed qualitatively. These findings not only provided novel empirical support for the longstanding theoretical predictions of Weiss [9] and Russell et al. [21], but they also indicated that classifying individuals as lonely based on a particular cut-off score is possibly misguided as such an approach fails to recognise naturally occurring subtypes of loneliness.

Based on the LCA results, approximately one-in-eight US adults aged 18-70 (12.4\%) were characterised by the simultaneously presence of social and emotional loneliness. This class had mean levels of psychological wellbeing, MDD, and GAD that were reflective of psychiatric morbidity. Additionally, approximately one-in-four US adults aged 18-70 (26.6\%) were characterised exclusively by the experience of emotional loneliness. This group of people, while less psychologically distressed than the 'social and emotional loneliness' class, were nonetheless characterised by mean levels of psychological wellbeing, MDD, and GAD that were also reflective of psychiatric morbidity. The combined proportion of individuals in these latent classes of loneliness who were characterised by clinically relevant levels of psychological distress was $39.0 \%$. This finding indicates that by recognising naturally occurring subtypes of loneliness, the number of people experiencing a form of loneliness that is likely to be of clinical relevance is more than double the number identified when loneliness is conceptualised as a unidimensional construct (39.0\% vs. $17.1 \%$ ).

Although another $8.2 \%$ of the population were characterised exclusively by the experience of social loneliness, individuals in this latent class were characterised by mental health scores reflective of healthy psychological functioning. Individuals characterised by 'social loneliness' had mental health scores that were not meaningfully different from individuals in the 'low loneliness' class. Our results show that when subtypes of loneliness are identified in a methodological rigorous manner, it is 'emotional' but not 'social' loneliness that is associated with poorer psychological health. These findings suggest that not all types of loneliness are necessarily detrimental to one's mental health. More importantly, these results indicate that the perception of inadequate close attachments to others is considerably more detrimental to one's mental health than the perception of inadequate social integration. To put it another way, it is the quality, not the quantity, of interpersonal connections that makes the difference when it comes to one's psychological health.

Support for the discriminant validity of the loneliness subtypes was found in relation to the specific correlates of class membership. For example, being single, divorced, or widowed increased the likelihood of belonging to the "emotional loneliness' class by nearly two-times, but had no association with membership of the 'social loneliness' class. Similarly, females were approximately two-times more likely than males to belong to the 'emotional loneliness' class, but no sex differences were evident in relation to membership of the 'social loneliness' class; findings that are generally consistent with prior observations [10,13]. Childhood traumatization was associated with 'emotional' but not 'social' loneliness, with every childhood traumatic experience increasing the odds of belonging to the "emotional loneliness' class by $28 \%$. It appears therefore that traumatization during childhood is associated with feelings of insufficient interpersonal attachments in later life. Childhood trauma has been demonstrated to disrupt healthy attachment relationships throughout life [50] and to lead to social withdrawal and social isolation [51]. It was interesting to note that childhood and adulthood trauma were independently associated with an increased likelihood of belonging to the 'social and emotional loneliness' class. The current study was the first to simultaneously assess the relationship between loneliness and both childhood and adulthood trauma, and our results indicated that traumatic exposure in these different developmental periods were positively associated with feelings of deficiencies in both social network size and intimate connections. Current results add to a growing literature attesting 
to the importance of trauma history in understanding the characteristic nature of the experience of loneliness [14-19].

Although distinguished by multiple factors, membership of the 'social', 'emotional', and 'social and emotional' loneliness classes was associated with younger age. These findings are consistent with the existing literature that loneliness follows a 'U-shaped distribution' of increasing levels of loneliness in early adulthood before declining through adulthood and then peaking again in older adulthood [5]. Given that this sample did not include individuals over the age of 70 , it is unsurprising that age was negatively correlated with all types of loneliness.

The importance of trauma history in the context of loneliness was further demonstrated by the results of the class-specific analyses. Amongst the 'low-loneliness' class, adulthood traumatization was significantly associated with poorer psychological wellbeing, MDD, and GAD. Of note, adulthood trauma was significantly associated with MDD and GAD for those characterised by 'social loneliness', whereas, childhood trauma was significantly associated with MDD, GAD, and psychological wellbeing for those characterised by 'emotional loneliness'. Our results show that not only are the loneliness subtypes differentially associated with childhood and adulthood trauma, but the relationship between mental health status and developmental timing of traumatic exposure is dependent upon the specific subtype of loneliness that one experiences. These findings support the value of considering different types of social/interpersonal clinical interventions depending on trauma history. Social interventions are likely to be of benefit to those with adult trauma; interpersonal/attachment interventions are likely to be of benefit to those with childhood trauma; and social and interpersonal interventions are likely to be of benefit to those with a history of both childhood and adulthood trauma.

A particularly curious finding was that the explanatory power of the regression models was highly dependent upon the type of loneliness being experienced, and, whether one considered positive or negative mental health indicators. Trauma history and demographic factors explained almost no variation in psychological wellbeing, MDD, and GAD scores for those in the 'low-loneliness' class (1-2\% of variance explained) and explained a higher percentage of variation in each mental health variable (11-17\% of variance explained) for those in the 'social loneliness' class. Furthermore, these variables explained a substantial level of variation in MDD and GAD scores for those individuals in both the 'emotional' (21\% and $25 \%$, respectively) and 'social and emotional' (27\% and 35\%, respectively) loneliness classes. However, the same variables accounted for very little variance in psychological wellbeing scores amongst the 'emotional' (8\%) and 'social and emotional' (6\%) loneliness classes. One might have expected that factors such as sex, age, relationship status, and traumatic history would contribute to an understanding of mental health variables irrespective of the type of loneliness one was characterised by; however, our results demonstrate that the explanatory power of these variables was highly dependent on (1) whether one was lonely or not, (2) the type of loneliness that one was experiencing, and (3) whether indicators of positive or negative mental health were being considered. These results have important implications for how clinical researchers should think about how loneliness might moderate the relationship between well recognised risk-factors and mental health.

The nationally representative nature of the sample, along with the application of sophisticated latent variable modelling techniques to identify subtypes of loneliness and their relationship to a variety of risk-factors and mental health variables, overcomes many of the limitations of the existing literature in this area. However, the current study is not without its limitations. For example, old age is a period of life where loneliness increases however the current sample did not include any members of the population over the age of 70. It will be important to replicate this study amongst cohorts of the population that include persons over the age of 70. Additionally, the study findings are reflective of the US adult population, and therefore, the cross-cultural validity of these findings is unknown. It will be particularly important to determine if current findings replicate in culturally distinct populations. Finally, the cross-sectional nature of the study precludes any inferences regarding the predictive relationships between traumatic exposure and loneliness class membership, or, the predictive relationships between trauma history and mental health status dependent upon one's loneliness subtype.

In sum, the current study provides empirical support for the existence of distinct subtypes of loneliness. Our study findings highlight the importance of recognising subtypes of loneliness given the considerable variation in mental health status, the unique associations with demographic and traumagenic variables, and the influence that these subtypes of loneliness have on the associations between established risk-factors (e.g., childhood and adulthood traumatization) and mental health status. The current findings also revealed that as a result of recognizing the naturally occurring subtypes of loneliness, the number of US adults aged 18-70 who experienced loneliness of a type that is associated with serious mental health difficulties is more than twice as high as the figure obtained when loneliness is treated as a unidimensional construct. Finally, our findings revealed that the perception of reduced quality, not quantity, of interpersonal relationships was associated with poor psychological health. From a societal perspective, and in the interests of reducing the burden of psychological distress, efforts should be made to enhance the quality of social connections as opposed to promoting the virtues of larger social networks. 
Author contributions $\mathrm{PH}, \mathrm{MS}, \mathrm{MC}$, and JMP developed the study concept. PH, MS, GM, and RF conducted the statistical analyses. JMP wrote the introduction. TK, FV, and MC contributed to the writing of the discussion. All authors reviewed, revised, and contributed to the writing of the final version of the manuscript. All authors have approved the final version of the paper for submission.

Funding This work was supported by the National Institutes of Mental Health (Grant number R01 MH08661).

\section{Compliance with ethical standards}

Conflict of interest On behalf of all authors, the corresponding author states that there is no conflict of interest.

\section{References}

1. Hunter D (2012) Loneliness: a public health issue. Perspect Public Health 132:153-153. https://doi.org/10.1177/1757913912449564

2. Cacioppo JT, Hawkley L, Thisted RA (2010) Perceived social isolation makes me sad: 5-year cross-lagged analyses of loneliness and depressive symptomatology in the Chicago health, aging, and social relations study. Psychol Aging 25:453-463. https://doi. org/10.1037/a0017216

3. Caspi A, Harrington H, Moffitt TE, Milne BJ, Poulton R (2006) Socially isolated children 20 years later: risk of cardiovascular disease. Arch Pediatr Adolesc Med 160:805-811. https://doi. org/10.1001/archpedi.160.8.805

4. Stickley A, Koyanagi A, Roberts B, Richardson E, Abbott P, Tumanov S, McKee M (2013) Loneliness: its correlates and association with health behaviours and outcomes in nine countries of the former Soviet Union. PLoS One 8:e67978. https://doi. org/10.1371/journal.pone.0067978

5. Lasgaard M, Friis K, Shevlin M (2016) "Where are all the lonely people?" A population-based study of high-risk groups across the life span. Soc Psychiatry Psychiatr Epidemiol 51:1373-1384. https://doi.org/10.1007/s00127-016-1279-3

6. Stravynski A, Boyer R (2001) Loneliness in relation to suicide ideation and parasuicide: a population-wide study. Suicide Life Threat Behav 31:32-40

7. Anderson OG (2010) Loneliness among older adults: a national survey of adults $45+$. AARP Research, Washington, DC. https:// doi.org/10.26419/res.00064.001

8. Sønderby LC, Wagoner B (2013) Loneliness: an integrative approach. J Integr Soc Sci 3:1-29

9. Weiss RS (1974) The provisions of social relationships. In: Rubin Z (ed) Doing unto others: Joining, molding, conforming, helping, loving. Prentice-Hall, Englewood Cliffs, pp 17-26

10. Gierveld JDJ, Van Tilburg T (2010) The De Jong Gierveld short scales for emotional and social loneliness: tested on data from 7 countries in the UN generations and gender surveys. Eur J Ageing 7:121-130. https://doi.org/10.1007/s10433-010-0144-6

11. Liu BS, Rook KS (2013) Emotional and social loneliness in later life: associations with positive versus negative social exchanges. J Soc Pers Relatsh 30:813-832. https://doi.org/10.1177/02654 07512471809

12. Dahlberg L, McKee KJ (2014) Correlates of social and emotional loneliness in older people: evidence from an English community study. Aging Ment Health 18:504-514. https://doi. org/10.1080/13607863.2013.856863

13. Dykstra PA, Fokkema T (2007) Social and emotional loneliness among divorced and married men and women: comparing the deficit and cognitive perspectives. Basic Appl Soc Psych 29:1-12. https://doi.org/10.1080/01973530701330843

14. van der Velden PG, Pijnappel B, van der Meulen E (2017) Potentially traumatic events have negative and positive effects on loneliness, depending on PTSD-symptom levels: evidence from a population-based prospective comparative study. Soc Psychiatry Psychiatr Epidemiol 53:1-12. https://doi.org/10.1007/s0012 7-017-1476-8

15. Cohen-Mansfield J, Shmotkin D, Goldberg S (2009) Loneliness in old age: longitudinal changes and their determinants in an Israeli sample. Int Psychogeriatr 21:1160-1170. https://doi.org/10.1017/ S1041610209990974

16. Gibson RL, Hartshorne TS (1996) Childhood sexual abuse and adult loneliness and network orientation. Child Abuse Negl 20:1087-1093

17. Merz EM, Jak S (2013) The long reach of childhood. Childhood experiences influence close relationships and loneliness across life. Adv Life Course Res 18:212-222. https://doi.org/10.1016/j. alcr.2013.05.002

18. Stein JY, Itzhaky L, Levi-Belz Y, Solomon Z (2017) Traumatization, loneliness, and suicidal ideation among Ex-POWs: a longitudinally assessed sequential mediation model. Front Psychiatry 8:281. https://doi.org/10.3389/fpsyt.2017.00281

19. Shevlin M, McElroy E, Murphy J (2015) Loneliness mediates the relationship between childhood trauma and adult psychopathology: evidence from the adult psychiatric morbidity survey. Soc Psychiatry Psychiatr Epidemiol 50:591-601. https://doi. org/10.1007/s00127-014-0951-8

20. DiTommaso E, Spinner B (1997) Social and emotional loneliness: a re-examination of Weiss' typology of loneliness. Pers Individ Differ 22:417-427. https://doi.org/10.1016/S0191-8869(96)00204 $-8$

21. Russell D, Cutrona CE, Rose J, Yurko K (1984) Social and emotional loneliness: an examination of Weiss's typology of loneliness. J Pers Soc Psychol 46:1313-1321

22. Peerenboom L, Collard RM, Naarding P, Comijs HC (2015) The association between depression and emotional and social loneliness in older persons and the influence of social support, cognitive functioning and personality: a cross-sectional study. J Affect Disord 182:26-31. https://doi.org/10.1016/j.jad.2015.04.033

23. Schnittger RI, Wherton J, Prendergast D, Lawlor BA (2012) Risk factors and mediating pathways of loneliness and social support in community-dwelling older adults. Aging Ment Health 16:335346. https://doi.org/10.1080/13607863.2011.629092

24. Drageset J, Espehaug B, Kirkevold M (2012) The impact of depression and sense of coherence on emotional and social loneliness among nursing home residents without cognitive impairment—a questionnaire survey. J Clin Nurs 21:965-974. https:// doi.org/10.1111/j.1365-2702.2011.03932.x

25. Shevlin M, Murphy S, Murphy J (2014) Adolescent loneliness and psychiatric morbidity in the general population: identifying "at risk" groups using latent class analysis. Nord J Psychiatry 68:633-639. https://doi.org/10.3109/08039488.2014.907342

26. Russell DW (1996) UCLA loneliness scale (version 3): reliability, validity, and factor structure. J Pers Assess 66:20-40. https://doi. org/10.1207/s15327752jpa6601_2

27. de Jong Gierveld J, van Tilburg TG (2006) A 6-item scale for overall, emotional, and social loneliness: confirmatory tests on survey data. Res Aging 28:582-598. https://doi.org/10.1177/0164027506 289723

28. De Jong Gierveld J, Van Tilburg T (2010) The De Jong Gierveld short scales for emotional and social loneliness: tested on data from 7 countries in the UN generations and gender surveys. Eur J Ageing 7:121-130. https://doi.org/10.1007/s10433-010-0144-6

29. Shevlin M, Murphy S, Mallet J, Stringer M, Murphy J (2013) Adolescent loneliness and psychiatric morbidity in Northern Ireland. 
Br J Clin Psychology 52:230-234. https://doi.org/10.1111/ bjc. 12018

30. Weathers FW, Blake DD, Schnurr PP, Kaloupek DG, Marx BP, Keane TM (2013) The life events checklist for DSM-5 (LEC-5). Instrument available from the National Center for PTSD at http:// www.ptsd.va.gov. Accessed 25 June 2017

31. Felitti VJ, Anda RF, Nordenberg D, Williamson DF, Spitz AM, Edwards V, et al. (1998) Relationship of childhood abuse and household dysfunction to many of the leading causes of death in adults. The Adverse childhood experiences (ACE) study. Am J Prev Med 14:245-258

32. World Health Organization: Regional Office for Europe (1998) Wellbeing measures in primary health care: the DepCare project. In: Consensus meeting, Stockholm

33. Topp CW, Østergaard SD, Søndergaard S, Bech P (2015) The WHO-5 well-being index: a systematic review of the literature. Psychother Psychosom 84:167-176. https://doi. org/10.1159/000376585

34. Awata S, Bech P, Koizumi Y, Seki T, Kuriyama S, Hozawa A, et al. (2007) Validity and utility of the Japanese version of the WHO-five well-being index in the context of detecting suicidal ideation in elderly community residents. Int Psychogeriatr 19:7788. https://doi.org/10.1017/S1041610206004212

35. Kroenke K, Strine TW, Spitzer RL, Williams JB, Berry JT, Mokdad AH (2009) The PHQ-8 as a measure of current depression in the general population. J Affect Disord 114:163-173. https://doi. org/10.1016/j.jad.2008.06.026

36. Spitzer RL, Kroenke K, Williams JB, Lowe B (2006) A brief measure for assessing generalized anxiety disorder: the GAD-7. Arch Intern Med 166:1092-1097. https://doi.org/10.1001/archi nte.166.10.1092

37. Manea L, Gilbody S, McMillan D (2015) A diagnostic metaanalysis of the Patient Health Questionnaire-9 (PHQ-9) algorithm scoring method as a screen for depression. Gen Hosp Psychiatry 37:67-75. https://doi.org/10.1016/j.genhosppsych.2014.09.009

38. Kertz S, Bigda-Peyton J, Bjorgvinsson T (2013) Validity of the generalized anxiety disorder-7 scale in an acute psychiatric sample. Clin Psychol Psychother 20:456-464. https://doi.org/10.1002/ cpp. 1802
39. Muthén LK, Muthén BO (2013) Mplus user's guide, 7th edn. Muthén \& Muthén, Los Angeles

40. Yuan KH, Bentler PM (2000) Three likelihood-based methods for mean and covariance structure analysis with nonnormal missing data. Sociol Methodol 30:165-200. https://doi. org/10.1111/0081-1750.00078

41. Akaike H (1987) Factor analysis and the AIC. Psychometrika $52: 317-332$

42. Schwartz G (1978) Estimating the dimension of a model. [Ann Stat 6:461-464

43. Sclove SL (1987) Application of model-selection criteria to some problems in multivariate analysis. Psychometrika 52:333-343

44. Lo Y, Mendell N, Rubin DB (2001) Testing the number of components in a normal mixture. Biometrika 88:767-778

45. Nylund KL, Asparouhov T, Muthén B (2007) Deciding on the number of classes in latent class analysis and growth mixture modeling. A Monte Carlo simulation study. Struct Equ Model 14:535-569. https://doi.org/10.1080/10705510701575396

46. Bakk Z, Vermunt JK (2016) Robustness of stepwise latent class modeling with continuous distal outcomes. Struct Equ Model 23:20-31. https://doi.org/10.1080/10705511.2014.955104

47. Bakk Z, Tekle FB, Vermunt JK (2013) Estimating the association between latent class membership and external variables using bias adjusted three-step approaches. In: Liao TF (ed) Sociological methodology. SAGE Publications, Thousand Oaks

48. Vermunt J (2010) Latent class modeling with covariates: two improved three-step approaches. Political Anal 18:450-469

49. Asparouhov T, Muthén B (2014) Auxiliary variables in mixture modelling: using the $\mathrm{BCH}$ method in Mplus to estimate a distal outcome model and an arbitrary secondary model. Stat Model. https://www.statmodel.com/download/asparouhov_muthen_2014. pdf. Accessed 25 June 2017

50. Pearce J, Simpson J, Berry K, Bucci S, Moskowitz A, Varese F (2017) Attachment and dissociation as mediators of the link between childhood trauma and psychotic experiences. Clin Psychol Psychother 24:1304-1312. https://doi.org/10.1002/cpp.2100

51. Walsh K, Fortier MA, DiLillo D (2010) Adult coping with childhood sexual abuse: a theoretical and empirical review. Aggress Violent Behav 15:1-13. https://doi.org/10.1016/j.avb.2009.06.009 